\title{
Sabrina Müller und Jasmine Suhner Eine Frage der Relation. Praktisch-theologische Annäherungen an die Frage nach Irrtum und Erkenntnis
}

Einseitige Wahrheit ist die ergiebigste Quelle des Irrtums. Johann Caspar Lavater

Wenn Menschen gefragt werden, was sie meinen, wenn sie von Erkenntnis oder Irrtum reden, erzählen sie meist Geschichten. Eigene Erfahrungen oder jene anderer. Alte und archetypische Geschichten ebenso wie aktuelle. Nicht nur das individuelle Gedächtnis beherbergt unzählige solcher Narrative, sondern auch das kulturelle und soziale Gedächtnis.

Ein wesentlicher Teil theologischer Forschung besteht darin, solche Geschichten zu interpretieren: aktuelle Geschichten, die als unmittelbare Erfahrungen und Erinnerungen im individuellen Gedächtnis verankert sind und durch empirische Forschung zugänglich gemacht werden; alte oder archetypische Geschichten, die durch systematische und hermeneutische Forschung erschlossen werden. Als im kulturellen Gedächtnis verankerte Erfahrungen fungieren letztere als Mythen der Kultur. Ihnen kann sich der einzelne Mensch nicht entziehen: Sie markieren in spezifischer Weise Orientierungspunkte, geben mögliche Leitlinien für die individuelle und gemeinschaftliche Reflexion von Denk- und Handlungsweisen vor. Stets geht es um Geschichten und Denkfiguren, die als ausgelegte zugleich den Menschen auslegen - und in diesem Zirkel auch für den heutigen Menschen zu relevanten anthropologischen Erkenntnissen führen. 
An den Anfang dieses Essays sei deshalb eine Geschichte gestellt. Eine archetypische Erzählung, die auf menschlicher Erkenntnis über den Menschen gründet und zugleich die menschliche Erkenntnis- und Irrtumsfähigkeit selbst zum Thema hat: ${ }^{1}$ die Schöpfungsgeschichte, ein Parademythos menschlichen Strebens nach Erkenntnis, der in den Grundzügen vielen bekannt ist. ${ }^{2}$

Und der Herr, Gott, liess aus dem Erdboden allerlei Bäume wachsen, begehrenswert anzusehen und gut zu essen, und den Baum des Lebens mitten im Garten und den Baum der Erkenntnis von Gut und Böse. Und der Herr, Gott, nahm den Menschen und setzte ihn in den Garten Eden, damit er ihn bebaute und bewahrte. Und der Herr, Gott, gebot dem Menschen und sprach: Von allen Bäumen des Gartens darfst du essen. Vom Baum der Erkenntnis von Gut und Böse aber, von dem darfst du nicht essen, denn sobald du davon isst, musst du sterben.

Dennoch ass der Mensch vom Baum der Erkenntnis. Die Reaktion Gottes wird folgendermassen beschrieben:

Und der Herr, Gott, sprach: Sieh, der Mensch ist geworden wie unsereiner, dass er Gut und Böse erkennt.

Kein vernünftiger Theologe, keine vernünftige Theologin glaubt, dass Adam und Eva die ersten Menschen waren oder dass es ein Paradies mit zwei Bäumen gegeben und der Mensch sich darin existentiell geirrt hätte. Diese biblische Geschichte ist zu verstehen, wie Legenden, Mythen oder Träume zu verstehen sind: Sie haben ihre Wahrheit, aber diese Wahrheit ist keine historische oder naturwissenschaftliche, sondern eine gleichsam mythopoetische, eine anthropologische Wahrheit des Mensch-Seins. Als solche betrifft sie den Menschen hier und heute.

Jegliche Vermutung - wie sie in manchen Öffentlichkeiten zuweilen geäussert wird -, die akademische Theologie befasse sich im

1 Vgl. Stephen Greenblatt: Die Geschichte von Adam und Eva. Der mächtigste Mythos der Menschheit, München 2018; Carel von Schaik, Kai Michel: Das Tagebuch der Menschheit. Was die Bibel über unsere Evolution verrät, Hamburg 2017.

2 Gen 2,9.15-17 u. 3,22. 
Rahmen ihrer hermeneutischen Tätigkeit in einem leichtgläubigen biblizistischen Sinne mit Geschichten und sei nicht fähig, zu «richtiger», wissenschaftlicher Erkenntnis zu gelangen, ${ }^{3}$ ist insofern als Irrtum zu bezeichnen. Konkret im Blick auf diesen Schöpfungsmythos: Jegliche Vermutung, die Theologie irre in ihrem Wahrheitsanspruch, ist gleichsam als Irrtum über diesen Irrtum - über die Tätigkeit vernünftiger Theologie im Allgemeinen und über die Rezeptionsgeschichte dieses biblischen Mythos im Spezifischen - zu enttarnen.

Umso mehr stellt sich die Frage, inwiefern dieses archetypische Narrativ zu für den heutigen Menschen relevanten Erkenntnissen über den Menschen führen und zum heutigen Diskurs von Irrtum und Erkenntnis des Menschen einen Beitrag leisten kann.

Vom Mythos über Erkenntnis zur Hermeneutik der Erkenntnis

Nicht aus sprachwissenschaftlichen, sondern aus theologischen Gründen ist die exegetische Forschung zunächst gehalten, die Art und Weise der Verwendung - und damit Bedeutung ${ }^{4}$ - des Wortfelds «erkennen» in seinem Kontext wahrzunehmen. Die Schöpfungserzählung verwendet das hebräische Verb ידע beziehungsweise, in der Septuaginta, das griechische Verb $\gamma \iota v \omega ́ \sigma \kappa \omega .{ }^{5}$ Beide Wörter werden

3 Vgl. Richard Dawkins: Die Schöpfungslüge. Warum Darwin recht hat, Berlin 2010; Christopher Hitchens: Der Herr ist kein Hirte. Wie Religion die Welt vergiftet, München 2009.

«Die Bedeutung des Wortes ist sein Gebrauch in der Sprache.» Ludwig Wittgenstein: Philosophische Untersuchungen (1953), in: Werkausgabe in acht Bänden, I, Berlin 1984, 225-280, § 43.

Dabei gilt es im Umgang mit den Bedeutungsaspekten der Begriffe ידע und $\gamma\llcorner\nu \omega \sigma \kappa \omega$ Vorsicht walten zu lassen und eine voreilige Gleichsetzung mit den Nuancen des deutschen Begriffs «erkennen» zu vermeiden. Um der spezifischen Semantik der biblischen Begrifflichkeiten Rechnung zu tragen, müssten die Aspekte des deutschen Begriffs der «Erkenntnis» untersucht werden, was im Rahmen dieses Essays nicht geleistet werden kann; verwiesen sei beispielsweise auf Franz von Kutschera: Grundfragen der Erkenntnistheorie, Berlin 1981. 


\section{Sabrina Müller und Jasmine Suhner: Eine Frage der Relation}

mit einer grossen Bandbreite an sich überschneidenden Bedeutungen verwendet.

Inhaltlich steht im Fokus der Schöpfungsgeschichte die Fähigkeit zur objektiven Erkenntnis: die «gottgleiche Unterscheidungsfähigkeit», die Differenzkompetenz im Blick auf Gut und Böse. Diese wirklichkeitsordnende und damit lebensorientierende Fähigkeit betont die biblische Tradition als fundamental. Sie schildert den Erwerb dieser grundlegenden kulturellen Erkenntnisfähigkeit des Menschen als ein Vermögen, das der Mensch nicht erworben, sondern sich von Gott erschlichen hat.

Wenn nun vom Baum der Erkenntnis von Gut und Böse die Rede ist, dann steht damit keineswegs primär das sittlich Gute oder Böse im Blick. Die Frucht des Erkenntnisbaums steht für die spezifische Teilhabe an der göttlichen Erkenntnis- und Unterscheidungsfähigkeit, das heisst, es geht um die daraus resultierende Schöpfungsfähigkeit des Menschen. Implizit enthält diese Bestimmung von Erkenntnis auch eine Bestimmung der entsprechenden Kontrastbezeichnung, des Irrtums: Irrtum ist der Einbruch, die Unterbrechung dieser Unterscheidungs- und damit der Schöpfungstätigkeit. Und Phänomene unter diesen beiden begrifflichen Polen zu beschreiben, ist eine fundamentale Weise menschlicher Weltorientierung und Lebensgestaltung.

Folgender Sachverhalt ist nun für beide Begriffsverständnisse relevant: Erkenntnis - und damit auch Irrtum - ist hier explizit nicht deskriptiv zu verstehen. Erkenntnisorientierte Tätigkeit zeigt sich stets als Beziehungsgeschehen, als nur in Bezug auf etwas möglich: auf den Menschen, auf den jeweiligen Kontext usw. ${ }^{6}$ Auch in seiner objektiven, kognitiven Dimension eignet ידע die Bedeutung von «anerkennen», «einsehen», also unabdingbar das

6 So zeigt etwa Willy Schottroff differenziert auf, dass sich durch alle Bedeutungen des hebräischen ידע hindurch ein relationaler Aspekt des «Erkennens» festhalten lässt. Vgl. Heinzpeter Hempelmann: Die Wirklichkeit Gottes. Theologische Wissenschaft im Diskurs mit Wissenschaftstheorie, Sprachphilosophie und Hermeneutik, Göttingen 2015, 46. 
Moment der Erfahrung von Geschichte, und damit ein affektiver, persönlicher Bezug. ${ }^{7}$ Damit wird zum einen auf eine unaufhebbare Ambivalenz der Phänomene hingewiesen, die die ihnen zugeschriebene Eigenschaft stets in Beziehung auf etwas oder jemanden erhalten. ${ }^{8}$ Erkenntnis impliziert insofern, zum anderen, unabdingbar eine Entscheidung darüber, wie der Mensch sich selbst versteht. Sie bedingt Selbsterkenntnis. Ohne ein solches vorgängiges Wissen darum, wer der Mensch ist, lässt sich nicht entscheiden, ob ein Sachverhalt als Erkenntnis oder Irrtum, als gut oder böse, als nützlich oder schädlich zu bewerten ist.

Die Frage nach dem Selbstverständnis des Menschen, nach seiner Selbstverortung ist aus einer transdisziplinären Forschungsperspektive relevant. Im spezifisch theologischen Sinn spielt die Schöpfungsgeschichte die Überzeugung ein, dass der Mensch ohne das Bewusstsein um seinen Gottesbezug, ohne das Wissen um die Differenz zwischen Schöpfer und Geschöpf das Bewusstsein um die eigenen Grenzen und Begrenztheiten und damit das, was ihn erst zum Menschen macht, ausblendet und wirklicher Erkenntnismöglichkeit zum Vornherein eine Absage erteilt.

$7 \quad$ Vgl. etwa Ez 14,23; 17,24 oder Joel 4,17. Zur Diskussion vgl. Walther Zimmerli: Erkenntnis Gottes nach dem Buche Ezechiel, in: Gottes Offenbarung, Gesammelte Aufsätze zum Alten Testament, München 1963, 41-119; H. Hempelmann: Die Wirklichkeit Gottes, 45-68.

Auch die 90 Belege für das entsprechende Substantiv דעת («Erkenntnis») verweisen in keiner Weise auf ein sich in distanzierter Weise formierendes Wissen. Vergleichbares, wenn auch nicht Gleiches, lässt sich nicht zuletzt auch im Blick auf das biblische Ziel von Weisheit festhalten: Um Erkenntnis zu gewinnen, müssen sich die Weisheitssuchenden auf einen Weg begeben, sich also selbst in Bewegung und sich in eine bestimmte Beziehung setzen. Vgl. Gerhard von Rad: Weisheit in Israel, Neukirchen-Vluyn 1970, 92. In diesem Zusammenhang ist die Frage zu stellen, inwiefern einem solchen sprachlichen Befund Aussagekraft zuzugestehen ist. Vor der Annahme bewusster Sprachführung der Autoren dieser zum kulturellen Gedächtnis gewordenen Geschichten sind solche sprachlichen Befunde zumindest nicht von den damit bezeichneten Inhalten zu trennen und insofern als relevant für den Gehalt dieser Geschichten zu betrachten. 


\section{Sabrina Müller und Jasmine Suhner: Eine Frage der Relation}

Wird Erkenntnis als Beziehungsgeschehen und Perspektivenfrage verstanden, steht die Kontrastlinie zwischen Erkenntnis und Irrtum keineswegs unveränderlich fest; sie ist vielmehr Veränderungsprozessen unterworfen, in denen der Bereich zwischen beiden Orientierungspolen permanent neu definiert wird. ${ }^{9}$ Das lässt sich bereits angesichts der Tatsache feststellen, dass entsprechende Unterscheidungs- und Erkenntnisleistungen auch innerhalb derselben sozialen Gruppen - etwa innerhalb eines intradisziplinären wissenschafts- und erkenntnistheoretischen Diskurses - selten gänzlich homogen sind, sondern sich fortlaufend Konstellationen und Fragen ergeben, deren Beurteilung eine Dynamik der entsprechenden Differenzierungsgrenze nach sich zieht. ${ }^{10}$ Funktional erübrigt sich jedoch die Kontrastfigur von Erkenntnis und Irrtum damit nicht: Denn gerade um sie für nötig oder unnötig zu erklären, muss man sie als Unterscheidung wiederum hinzuziehen.

Insgesamt resultiert jegliche Beschreibung von Phänomenen unter dem Gesichtspunkt von Erkenntnis und Irrtum in einer weit komplexeren und weniger eindeutigen Weltsicht, als dies im Sinne unbezweifelbarer Forschungsergebnisse und Lebensorientierung zu wünschen ist - und zwar sowohl auf der Ebene der Unterscheidung selbst als auch auf jener ihrer Anwendung zur Orientierung, das heisst im Blick auf konkrete Lebensphänomene.

Von hier aus bedürfen gerade diese beiden Kontexte - existentielle Weltzugänge wie anthropologisch bedingte Forschungsbemühungen - der besonderen Einübung in die kritische Scheidung

9 Mit einer solchen Betonung von Erkenntnis als Beziehungsgeschehen wird jenen Erkenntnisbemühungen eine Absage erteilt, die für die Möglichkeit wahrer Erkenntnis durch die Vermeidung jeglicher sinnlichen Wahrnehmung beziehungsweise den Rückzug auf das reine Denken plädieren, so René Descartes: Meditationes de prima philosophia. Meditationen über die Grundlagen der Philosophie (1641), hg. von Hans Günter Zekl, Hamburg 21977, 22.

10 Dies lässt sich exemplarisch nachzeichnen an den Verschiebungen moralischer Bewertungen, die sich ebenfalls häufig zwischen zwei Polen - gut und böse, nützlich und schädlich u.ä. - bewegen. Vgl. Ingolf U. Dalferth: Das Böse. Essay über die Denkform des Unbegreiflichen, Tübingen 2006, 77. 


\section{Sabrina Müller und Jasmine Suhner: Eine Frage der Relation}

zwischen dem Wahren und dem Falschen, dem Erkannten und Nicht-Erkennbaren. Hier hat die akademische Theologie in einer sich über die Jahrhunderte zunehmend ausdifferenzierenden hermeneutischen Forschung ein spezifisches und reflexives Vermögen und ein breites methodisches Instrumentarium entwickelt.

Wie sind theologische Tätigkeit und Erkenntnisfähigkeit zu denken?

In Bezug auf den Schöpfungsmythos und sein Verständnis ist, zumal aus reformiert-theologischer Sicht, eine programmatische Kontextualität einzufordern. Die Theologie muss sowohl empirisch als auch hermeneutisch ihre eigenen normativen Prämissen stets in eine Beziehung zur Lebenswelt setzen und einer permanenten kritischen Prüfung unterziehen. Damit stellen weder die Ausgangspunkte noch die Gegenstände noch das Ziel theologischer Reflexionsbemühungen etwas Statisches dar, über das eine möglichst objektive Lehre vermittelt werden kann oder soll. Gerade die biblisch-historischen Grundbezüge von Theologie sind so divers, dass um Erkenntnis, Wahrheit und Irrtum seit je gerungen werden muss: dies gerade oft auch gegen den offiziellen Deutungsmachtanspruch der den Glauben repräsentierenden Institutionen und ihrer akademisch-orthodoxen Lehre(r). Insofern ist Theologie seit ihren ersten Anfängen - und dies gilt sich nicht nur für die christliche Theologie - nicht etwa primär eine feststellende und ergebnissichernde Wissenschaft, sondern zugleich und ebenso ein dynamisches «doing theology», ${ }^{11}$ ein praxisbezogenes hermeneutisches

11 Diese Begrifflichkeit hat durchaus, vereinzelt, bereits vor einigen Jahrzehnten in Ansätzen Fuss gefasst, vgl. David Tracy, John. B. Cobb: Talking about God. Doing Theology in the Context of Modern Pluralism, San Francisco 1984. Verstärkt im Fokus einschlägiger Debatten steht sie erst seit wenigen Jahren, vgl. etwa John Parratt (ed.): A Guide to Doing Theology, Minneapolis 2015, 101; John W. de Gruchy: From Political to Public 
Reflektieren. Solches Reflektieren steht im Dialog mit dem, was oben als Geschichten bezeichnet worden ist: mit historischen Quellen sowie vielfältigen Erfahrungen. Im weiteren Sinn zählen hierzu auch Analysen von Sprachsystemen, gegenwärtige Entwicklungen, theologische und andere Forschungsergebnisse, öffentliche und individuelle Problemstellungen, insbesondere in ihrer Diversität gelebte Religion in Politik und Gesellschaft im digitalen und analogen Raum. ${ }^{12}$ Theologische Forschung gewinnt insofern ihr spezifisches, sie von anderen Forschungskulturen unterscheidendes Profil nicht in erster Linie durch ihre Methoden oder die Sachverhalte, die sie analysiert und reflektiert, sondern dadurch, dass und wie sie diese Phänomene zur Sprache bringt. Sie bleibt gleichsam stets Theologie, indem sie sich dem Sprechen im Horizont des «etsi Deus daretur», ${ }^{13}$ also der Möglichkeit transzendenter Existenz, verpflichtet. ${ }^{14}$

In dieser spezifischen Weise spricht die Theologie fundamentale menschliche Orientierungsweisen und -fragen an. Sie ist dadurch konstruktiv-kritische Hermeneutik existentieller Dimensionen des Menschseins, ${ }^{15}$ die gerade jene gesellschaftlichen und wissenschaft-

Theologies. The Role of Theology in Public Life in South Africa, in: William F. Storrar, Andrew R. Morton (eds.): Public Theology for the $21^{\text {st }}$ Century. Essays in honour of Duncan Forrester, London, New York 2004, 45-62, hier 53.

12 Thorsten Meireis: Überarbeitung der Antrittsvorlesung an der Theologischen Fakultät der Humboldt-Universität zu Berlin vom 21.06.2017, gehalten im Rahmen der Eröffnungskonferenz des Berlin Institute for Public Theology (21.-22.06.2017); Sabrina Müller: Gelebte Theologie. Impulse für eine Pastoraltheologie des Empowerments, Zürich 2019. Vgl. Ingolf U. Dalferth: God first. Die reformatorische Revolution der christlichen Denkungsart, Leipzig 2018, 52.

Vgl. Jasmine Suhner: Menschenrechtspädagogische Bezugsfelder religiöser Bildung. Theologisch-religionspädagogische Grundsatzüberlegungen, Konkretisierungen und Perspektiven (Dissertationsmanuskript), Zürich 2019, 129-145.

15 «Revelation is the manifestation of what concerns us ultimately. The mystery which is revealed is of ultimate concern to us because it is the ground of our being.» Paul Tillich: Systematic Theology, I, Chicago 1973, 110. «Es gibt nichts, was in diesem Horizont nicht in ihren [sc. der Theologie] 
lichen Normativitäten hinterfragt, die den Menschen auf eindimensionale anthropologische Konzepte und Engführungen reduzieren wollen. In dieser Funktion kann die Theologie katalytische Wirkungen entfalten, indem sie - gerade auch religionsbezogene - Erfahrungen, die für das Individuum als intuitiver Wissens- und Erkenntnisgewinn fungieren, ${ }^{16}$ wahrnimmt, distanzierend herausstellt, nach einer Sprache für diese Erfahrungen sucht und sie kritisch auf lebensfördernde und lebenshindernde Mechanismen prüft. ${ }^{17}$ Sie fragt darüber hinaus nicht zuletzt - empirische und hermeneutische Tätigkeit auch überschreitend - danach, was spezifische Erfahrungen und deren Versprachlichungen über das Leben, über das Bild von Gesellschaft und Mensch aussagen. ${ }^{18}$ Als Unternehmung kritischer

Bereich fiele - auch wenn nicht alles in derselben Weise relevant ist für das, was sie erkundet.» Ingolf U. Dalferth: «Mit Gott kommt alles in den Blick», https: / / landeskirchenforum.ch/d3-vom-wert-der-theologie (abgerufen am 31.1.2019).

16 Vgl. die entsprechenden Ausführungen zum individuellen intuitiven Erkenntnisgewinn weiter unten.

17 Vgl. Sabrina Müller: How ordinary moments become religious experiences: a process-related practical theological perspective, in: Ulrich Riegel, EvaMaria Leven, Daniel Fleming: Religious Experience and Experiencing Religion in Religious Education, Münster 2018, 79-96. Um dies exemplarisch anzudenken: Wo - von welcher Seite auch immer behauptet wird, dass diese oder jene Erkenntnis oder Haltung gerechtfertigt ist, wird aus unterschiedlichen theologischen Perspektiven gefragt, ob die Erkenntnis oder Haltung wahr, und wenn ja, ob sie gut und inwiefern sie gerechtfertigt ist. Wo eine Haltung oder Handlung als gut oder gerecht charakterisiert wird, fragen die theologische Ethik und die Praktische Theologie entsprechend, nach welchen Standards dies geschehen kann, und ob die Behauptung über die Haltung oder Handlung wirklich angemessen ist. Die Theologie beteiligt sich auf diese Weise kontinuierlich am Gespräch über die Normen des Erkennens und Handelns. Dies lässt sich biblisch untermauern: Zum biblisch-christlichen Selbstverständnis zählt wesentlich der Auftrag zur Verkündigung des Evangeliums, zu der auch Bildungsverantwortung gehört. Gerade «jesuanisch» verstanden - erinnert sei daran, dass, unseres Erachtens durchaus programmatisch, Jesus selbst seine Lehre nicht verschriftlicht hat - kann Bildung kein statisches, vermittelndes Prinzip darstellen, sondern 
Provenienz intendiert sie umfassend die Kritik und Hinterfragung von unreflektierten oder unfreien Lebenshaltungen, Denk- oder Sprechweisen. ${ }^{19}$

Auf diese Weise nimmt Theologie gerade die Erkenntnisfähigkeit des Individuums ernst und reduziert dessen religiöse Erlebenswelt weder auf rein funktionale noch substantielle Religionskonzeptionen. Sowohl ein rein funktionaler Zugang als auch ein rein substantieller Zugang würden die Dignität und die Qualität aktueller menschlicher Erfahrungen in Frage stellen und dem kreativ-interpretativen Erkenntnisprozess kaum Rechnung tragen. ${ }^{20}$ Insofern ist nur eine diskursive, interpretative Bestimmung religiöser Erkenntnisfähigkeit sachangemessen. ${ }^{21}$ Dieser eignet der Vorteil, dass religiöse Erkenntnisfähigkeit deutungsoffen und veränderbar für Irrtum und Erkenntnis bleibt. In anthropologischer Perspektive wäre hier etwa auf Paul Ricœur zu verweisen, der den Menschen als hermeneutisches Wesen versteht, das immerzu darum bemüht ist, seine Erfahrungen zu interpretieren und in einen Sinnzusammenhang zu stellen. ${ }^{22}$

Die Kombination eines solchen weiten Theologiebegriffs und diskursiven Religionsverständnisses stellt ein plurales Diskurskonzept für Irrtum und Erkenntnis zur Verfügung. Darin werden

basiert grundlegend auf einem kommunikativen Modus religionspädagogischen Handelns.

19 Zum Begriff der «semiotischen Autonomie» vgl. Michael Hampe: Historische Einheit und semiotische Autonomie. Anthropologische Implikationen der Metaphysik von Peirce, in: ders.: Erkenntnis und Praxis, Berlin 2006, 53-75.

20 Vgl. etwa Heinz Streib zur Einseitigkeit eines funktionalen Zugangs: Eine rein funktionale Unterbestimmung von Religion führt zu «Einseitigkeit, wenn Religion auf ihre Leistung für das Subjekt oder die Gesellschaft beschränkt wird» Heinz Streib, Carsten Gennerich: Jugend und Religion. Bestandsaufnahmen, Analysen und Fallstudien zur Religiosität Jugendlicher, Weinheim 2011, 17.

21 Vgl. ebd., 14.

22 Paul Ricœur: Der Konflikt der Interpretationen. Ausgewählte Aufsätze (1960-1969), hg. von Daniel Creutz, Hans-Helmut Gander, Freiburg i.Br., München 2010, 23-47. 
nationale und religiöse Grenzen überschritten und kolonialistische sowie patriarchale Macht- und Deutungsansprüche in Frage gestellt. Dies gerade in kritischer Absetzung von der eigenen Herkunft und Wissenschaftsgeschichte. In dieser Art des Erkenntnisstrebens kann Theologie auch nicht linear von Feststellung zu Feststellung schreiten. Der jeweils aktuelle theologische Erkenntnisstand zeigt sich nicht lediglich als die Weiterentwicklung vergangener Erkenntnisse oder als Überwindung vergangener Irrtümer, sondern stets als spezifische Reaktion auf die Vergangenheit und die Gegenwart aufgrund der individuellen und öffentlichen Erfahrungen. ${ }^{23}$ Theologischer Erkenntnisgewinn zielt damit nicht auf die Produktion von Normativität, auf ein vereinfachendes Gegenüberstellen von Erkenntnis und Irrtum ab, auch nicht primär auf das richtige Urteil im Unterschied zum falschen. Vielmehr intendiert er kritisches Wahrnehmen, Kommunizieren, Positionieren und Prüfen - und dabei durchaus auch eine persönliche Ansprache: als dynamisches Erinnern, aktuelles Erleben, Reflektieren und öffentliches Kommunizieren. ${ }^{24}$

Die akademische Theologie bringt gerade aufgrund ihrer Entwicklungsgeschichte eine besonders hohe Fähigkeit zu spezifischer und differenzierter Hermeneutik ein. Sie vermag der einzelnen theologisch sich bildenden oder (welt-)perspektivisch sich orientierenden Person zu einer entsprechenden Beobachtungs- und Deutungsmöglichkeit zu verhelfen. Zudem fördert sie individuelle und öffentliche Auseinandersetzungen mit und Kommunikation über Weltzugang und -deutung. ${ }^{25}$ Dabei «knüpft [sie] zwar stets an bestimmte Glau-

23 Vgl. J. Suhner: Menschenrechtspädagogische Bezugsfelder religiöser Bildung, 129-145.

24 Vgl. Laurie Green: Let's Do Theology. Resources for Contextual Theology, London, New York 2009.

25 Zur Bedeutung der Theologie und insbesondere der akademischen Theologie für die Bildung und Erhaltung demokratischer Gesellschaften vgl. Alfons Knoll: Spiritualität, die sich verstehen will. Überlegungen zur Theologie als Glaubenswissenschaft, in: Guido Bausenhard et al. (Hg.): Zukunft aus der Geschichte Gottes, Freiburg i.Br. 1998, 449-478. 
benstraditionen an, bleibt aber nicht auf diese beschränkt, sondern ist allen zugänglich, die von ihrer Vernunft auch im Bereich von Religion und Glaube öffentlich Gebrauch zu machen gewillt sind. Und sie stellt ihre Resultate öffentlich zur Diskussion, indem sie diese nicht autoritär, sondern argumentativ vertritt und die Geltung ihrer Einsichten nicht auf ihre Herkunft und Genese, sondern auf die Nachvollziehbarkeit und Überzeugungskraft ihrer Argumente gründet.» ${ }^{26}$

In ähnlichem Sinn ist jede im öffentlichen Raum tätige Wissenschaft herausgefordert, ihre Perspektive gleichsam immer wieder zu unterbrechen und ihre Erkenntnisse vom Gesichtspunkt des Anderen oder des Unterschiedes neu zu betrachten. Sowohl natur- als auch geisteswissenschaftliche Forschung lebt vom Glauben an die Möglichkeit des Erkenntnisgewinns. Pointiert gesagt: In gewissem Sinn steht zu Beginn jedes einzelnen Forschungsprozesses die Hoffnung, etwas Bestimmtes zu entdecken und zu erkennen. Ohne ein solches Für-Möglich-Halten wäre jeder Beginn von Forschung sinnlos. Die Form, Gestalt und der Gewinn der Erkenntnis unterscheiden sich zwar je nach Wissenschaftsfeld, eine grundsätzliche Leistung und Gemeinsamkeit findet sich aber in der Fähigkeit zu erkennen $^{27}$ - und in der Fähigkeit, auf solche Erkenntnis zu hoffen und dafür auch Umwege, Sackgassen und Irrtümer in Kauf zu nehmen.

Vgl. Ingolf U. Dalferth: Vor Gott gibt es keine Beobachter. Öffentlichkeit, Universität und Theologie, in: ders.: Gedeutete Gegenwart. Zur Wahrnehmung Gottes in den Erfahrungen der Zeit, Tübingen 1997, 53. Es gehört unumgänglich zum Wesen akademischer Theologie, sich aktiv auf das Mensch-Sein im jeweiligen Kontext einzulassen - und damit auch, die (Gedanken-)Räume der (akademischen) Räumlichkeiten immer wieder zu verlassen. Vgl. Bernd Schröder: Theologische Bildung im öffentlichen Raum, in: Thomas Schlag, Jasmine Suhner: Theologie als Herausforderung religiöser Bildung, Stuttgart 2017, 151-178. Auf nicht nur die pädagogischen, sondern auch religiösen und theologischen Deutungen solcher Bildungsfähigkeit beziehungsweise Bildsamkeit hat der Erziehungswissenschaftler Benner in jüngerer Zeit mehrfach hingewiesen. Vgl. Dietrich Benner: Bildung und Religion. Nur einem bildsamen Wesen kann ein Gott sich offenbaren, Paderborn 2014, 27. 
Einstein wird der einprägsame Satz zugeschrieben: «Nur, wer nicht sucht, ist vor Irrtum sicher». ${ }^{28}$ Umgekehrt formuliert: Wer nach Erkenntnis sucht, muss Irrtümer und Irrwege in Kauf nehmen. Insofern muss jegliches Streben nach Erkenntnis als multiperspektivischer Prozess verstanden werden, der sich stets auch selbst zu hinterfragen hat. Gerade die hermeneutisch geschulte Theologie erinnert daran, dass die Potentialität der Unterscheidung zwischen Irrtum und Erkenntnis im Vordergrund steht. Sie verweist auf die Frage, wie mit Irrtum und Erkenntnis umgegangen werden kann - und in Verantwortung woraufhin. Kurz: Sie sensibilisiert für unbedingte Achtsamkeit im Blick auf den kritisch analysierenden Umgang mit Deutungsmächten, Machtverhältnissen und anthropologischen Prämissen im öffentlichen und privaten Leben.

Solche hermeneutischen Erkenntnisbemühungen werden in der pluralisierten und individualisierten Gesellschaft nicht weniger, sondern mehr denn je gebraucht. Gerade angesichts der wuchernden Daten- und Informationsmenge sind hermeneutische Zugänge und Kritik notwendig. Sie klären die Welt, bevor sie sie erklären. Oder sie klären bereits vorliegende Erklärungen. Sie bieten Möglichkeiten, den Verlauf des Denkens und Handelns selbstbestimmt zu rahmen, damit diese konstruktiv und lebensfördernd werden. Die Erkenntnisfähigkeit des aufgeklärten und mündigen Individuums, das die Welt kritisch interpretiert, steht hier im Zentrum. ${ }^{29}$ Theologisch formuliert: Es geht um den Blick weg von der Fülle hin zur Erfüllung - in Wissenschaft, Gesellschaft und individueller Lebensgestaltung. Auch in einer pluralisierten und individualisierten Gesellschaft fragen die Menschen danach, wie leben, wissen, lieben und Tod Sinn ergeben, weshalb ihnen Leid widerfährt oder wie sich berührende Momente verewigen lassen. Sie stellen also Fragen, die religiös gedeutet werden können. ${ }^{30}$ Hieran ändert auch die zunehmende

Alice Calaprice: Einstein sagt: Zitate, Einfälle, Gedanken, München 2007, 147. Vgl. S. Müller: Gelebte Theologie, 28-30.

Vgl. Hans Joas: Braucht der Mensch Religion? Über Erfahrungen der Selbsttranszendenz, Freiburg i.Br. 2004; ders.: Glaube als Option. Zukunfts- 
Pluralisierung von Lebensstilen, ${ }^{31}$ die Identitäts- und Sinnfindung immer mehr zur persönlichen Orientierungsleistung werden lässt, nichts. Vor dem Hintergrund des gesellschaftlichen Wandels, infolge dessen die Big Questions nicht mehr durch einen Sozialverband oder Sippenstrukturen beantwortet werden, ${ }^{32}$ unterliegt Identität vielmehr einem kontinuierlichen Konstruktionsprozess. ${ }^{33}$ So ist vom Einzelnen ein permanenter persönlicher Abgleich von Erkenntnis, Selbsterkenntnis und Selbstgestaltung gefordert. ${ }^{34}$

Gerade die jeweils persönlichen religiös gedeuteten Erfahrungen nehmen im Leben vieler Menschen eine Form des dauerhaften Erkenntnisgewinns an. Diese besitzen «eine zwar anders geartete, aber dennoch eigenständige Objektivität». ${ }^{35}$ In dieser Weise sich bildende religiöse Überzeugungen werden vom Individuum als «Wissenszuwachs angesehen, anhand dessen gehandelt und Leben gestaltet [wird]. Diese Normativität wird jedoch persönlich kon-

möglichkeiten des Christentums, Freiburg i.Br. 2012; Ann Taves: Finding and Articulating Meaning in Secular Experience, in: U. Riegel, E.-M. Leven, D. Fleming: Religious Experience, 13-22.

31 Vgl. Linda Woodhead: Religion and Change in Modern Britain, London, New York 2012.

Vgl. A. Taves: Finding and Articulating Meaning in Secular Experience, 13-22, hier 13-18.

33 Das Individuum ist selbst für die Beantwortung dieser Fragen zuständig und somit auch dafür, seinem Leben Richtung zu geben und ihm Sinn zu verleihen. So werden im Zuge der Individualisierung Glaubensüberzeugungen und Religiosität sowohl personalisiert (Gott wird personales Gegenüber), als auch von individuellen Erfahrungen abhängig gemacht. Vgl. Peter L. Berger: The Many Altars of Modernity. Toward a Paradigm for Religion in a Pluralist Age, Berlin, Boston 2014.

Vgl. Rolf Oerter, Leo Montada: Entwicklungspsychologie, Weinheim 2002, 292. Dabei ist die empfundene Identität «das subjektive Empfinden seiner eigenen Situation und seiner eigenen Kontinuität und Eigenart, das ein Individuum allmählich als ein Resultat seiner verschiedenen sozialen Erfahrungen erwirbt». Erwin Goffman: Stigma. Über Techniken der Bewältigung beschädigter Identität, Frankfurt a.M. 2010, 132. gen 1990. 
struiert, auf der Basis neuer Erfahrungen weiterentwickelt und sie

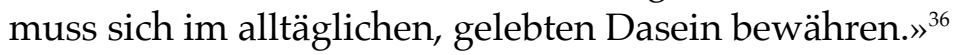

In diesem Feld neu sich anbahnender persönlicher Normativitäten, die aufeinander und auf wiederum öffentliche sowie im kulturellen Gedächtnis verankerte Normativitäten treffen, und in diesem komplexen Geflecht individueller und sich neu formierender gemeinschaftlicher Religiosität fragt insbesondere die Praktische Theologie nach der Entstehung, der gesellschaftlichen und sozialen Bedeutung sowie nach lebensfördernden oder lebenshindernden Aspekten gelebter Religion. ${ }^{37}$

\section{Von der Erkenntnisfähigkeit zur Erkenntnis des}

Erkanntwordenseins

Der dargelegte theologische Erkenntnisbegriff darf, gerade als kontextuell verstandener, weder allgemeine Gültigkeit noch absolute erkenntnistheoretische Relevanz beanspruchen. Diese Bestimmung steht gleichsam quer zu Erkenntnistheorien, die eine vom Subjekt gewonnene Erkenntnis erst als solche betrachten, wenn gerade die kontextuellen, relationalen Momente ausgeschlossen werden. ${ }^{38}$

Luther hat den Menschen als «homo incurvatus in se», als in sich verkrümmten Menschen, bestimmt und damit auf die Gefahr hingewiesen, dass der Mensch auf sich selbst zentriert die Welt zu erkennen und erklären suche, ohne ihr wirklich $\mathrm{zu}$ begegnen. ${ }^{39}$

S. Müller: Gelebte Theologie, 49.

Albrecht Grözinger, Georg Pfleiderer (Hg.): «Gelebte Religion» als Programmbegriff Systematischer und Praktischer Theologie, Zürich 2002; Thomas Schlag: Religionspädagogik als Lebenswissenschaft. Bildungstheoretische Vermessungen in weisheitlich-lebensdienlicher Perspektive, in: Zeitschrift für Theologie und Kirche 116 (2019) 228-249.

Hieraus resultieren dann auch entsprechend unterschiedliche Konsequenzen für die Ethik.

Martin Luther: Vorlesung über den Römerbrief (1515/16), hg. von Hans H. Borcherdt, Georg Merz, München ${ }^{3} 1965,187$. 
Erkennen bedeutet demgegenüber vor allem ein Sich-Beziehen auf etwas oder jemanden. ${ }^{40}$ An und für sich kann der Mensch keine Erkenntnis gewinnen - oder nur verzerrte. Die Theologie denkt «objektive» Erkenntnis gerade und nur dort als möglich, wo der Mensch nicht nur selbst erkennt, nicht nur Erkenntnissubjekt ist, sondern zugleich erkannt wird, gleichsam Objekt der Erkenntnis Gottes ist. ${ }^{41} \mathrm{Zu}$ seiner Erkenntnisfähigkeit gelangt der Mensch also aufgrund seines schon vorgängigen Erkanntwordenseins und Anerkanntseins durch Gott. Sein und Erkennen können nicht getrennt werden, weil «Erkennen» eine das kognitive Moment übersteigende, umfassende Form der Mit-Teilung eigener Existenz einschliessen kann. ${ }^{42}$ Zugleich ist diese Fähigkeit, theologisch gesprochen, Zusage und Geschenk an den Menschen. Durch sie wird das intrinsische Streben nach Erkenntnis genährt, das die existentielle Dimension von Irrtum und Erkenntnis in eine Resonanzbeziehung ${ }^{43}$ bringt mit dem, was das Individuum unbedingt angeht und betrifft - mit Tillich: dem «ultimate concern». ${ }^{44}$ So gesehen wird der Mensch durch den Schöpfungsmythos als Beziehungswesen entlarvt. Im Gegenüber erkennt er sich, wird selbst erkannt und kann Irrwege enttarnen. Das wahrhafte Wesen der Erkenntnis besteht eben darin, «das Bewusstsein seiner selbst aufzugeben, sich in einem anderen Selbst zu vergessen». ${ }^{45}$

Als theologisches Normativ kann deshalb nicht für eine Erkenntnis oder die Absenz von Irrtum plädiert, sondern muss die

$40 \quad$ Vgl. Martin Buber: Das Dialogische Prinzip, Gütersloh 2002.

41 Eine solche Betonung der Verflechtung objektiver Erkenntnis und der jeweiligen Perspektive kann, dies sei hier nur kurz angedeutet, nun gerade angesichts quantenphysikalischer Erkenntnisse auf interdisziplinäre Resonanz stossen.

42 Paul Tillich: Trennung und Einigung im Erkenntnisakt. Probleme einer Ontologie des Erkennens, in: Tillich-Auswahl, I: Das Neue Sein, hg. von Manfred Baumotte, Gütersloh 1980, 183-193 (GW IV 107-117).

43 Hartmut Rosa: Resonanz: Eine Soziologie der Weltbeziehung, Berlin 52016, 435-452.

$44 \quad$ Vgl. Paul Tillich: Dynamics of Faith, New York 1957, 1.

45 Georg Wilhelm Friedrich Hegel: Vorlesungen über die Ästhetik, Berlin, Weimar ${ }^{2} 1985,519$. 
Einsicht in die relationale Erkenntnisfähigkeit des Menschen benannt werden. Nicht Irrtum und Versagen ist des Menschen unwürdig, sondern die Verweigerung, sich mit einem Gegenüber am aktivkreativen Prozess von Irrtum und Erkenntnis, nach dem die Erkenntnisfähigkeit verlangt, zu beteiligen. Dies bedingt einen geübten, fehlerfreundlichen und humanen Umgang mit menschlichem Irrtum und Versagen. Letztlich können wir sowohl mit Irrtum als auch mit Erkenntnis nur im Bewusstsein um die Relationalität und Relativität menschlicher Erkenntnisfähigkeit angemessen umgehen - darin liegt unsere wissenschaftliche und menschliche Verantwortung.

conexus 2 (2019) 8-24

(C) 2019 Sabrina Müller und Jasmine Suhner. Dieser Beitrag darf im Rahmen der Lizenz CC BY-NC-ND 4.0 - Creative Commons: Namensnennung/ nicht kommerziell/keine Bearbeitungen - weiterverbreitet werden.

\section{(i) 8}

https: / / doi.org/10.24445/ conexus.2019.02.003

Dr. Sabrina Müller, Universität Zürich, Theologische Fakultät, Kirchgasse 9, 8001 Zürich

sabrina.mueller@theol.uzh.ch

Dr. des. Jasmine Suhner, Universität Zürich, Theologische Fakultät, Kirchgasse 9, 8001 Zürich

jasmine.suhner@theol.uzh.ch 